\title{
Identification and MPC Control of a Circulation Fluidized Bed Boiler Using an LPV Model
}

\author{
Jiangyin Huang *, Guoli Ji *, Yucai Zhu **, Wei Lin* \\ * Department of Automation, Xiamen University, Xiamen 361005 \\ China (Tel: 13860185962; e-mails:alrin476@gmail.com,glji@xmu.edu.cn) \\ ** Control Systems, Faculty of Electrical Engineering, Eindhoven University of Technology \\ The Netherlands (e-mail:y.zhu@tue.nl)
}

\begin{abstract}
This work studies the identification and control of circulation fluidized bed (CFB) boilers. The CFB boiler under investigation shows strong nonlinearity due to big changes of steam load. A linear parameter varying (LPV) model is used to represent the process dynamics and used in control. The steam flow is used as the working-point variable (scheduling variable) of LPV model. Multivariable plant tests were carried out and a LPV model and a linear model were identified. The identification results of the industrial CFB boiler show that the LPV model has much higher accuracy than the linear model. The simulation studies for the MPC control of the CFB boiler have been performed using both the LPV model and the linear model. The simulation results show that the MPC using the LPV model performs better than the MPC using the linear model.
\end{abstract}

Key words: circulation fluidized bed (CFB) boilers, identification, LPV model, MPC control

\section{INTRODUCTION}

Most industrial process systems are nonlinear. Because it is difficult to identify accurate models of nonlinear system, approximate linear models are used in most of industrial controllers, which may lead to lower control performance. It is important to develop simple and practical method for nonlinear process modelling and identification; and use these models for the control of nonlinear processes.

In nonlinear system identification, nonlinear AR(MA)X models, neural-networks models are often used. These models are complex in structure and difficult to compute numerically. Block-oriented nonlinear models such as Hammerstein models and Wiener models are simpler, but they can only model nonlinearity in static gains which is often too limiting in process control applications.

Linear parameter varying (LPV) models are used in process identification. In process control, there are several advantages using the LPV models: 1) they are simple to identify; 2) they can model both static and dynamic nonlinearities; and 3) they can take into account the process operation knowledge in selecting the working-point variables (scheduling variables). The terminology of LPV was first introduced by Shamma and Athans (1991) in the study of gain scheduling control. Applications of LPV (or gain scheduling) control have been reported in the control of electro-mechanical systems by Rugh and Shamma (2000). Several LPV model identification and control algorithms are developed in the literature. Among others, multiple-model approaches are studied in MurraySmith and Johansen (1997); deterministic-stochastic subspace approach is studied in Santos (2007); and orthonormal basis functions are used in Tóth et. al. (2009). Zhu and Xu (2008) proposed an LPV model in the form of blended linear models; Zhu and Ji (2009) proposed an LPV model as another form of blended linear models.

In this work, we take circulation fluidized bed (CFB) boiler as the research object and put forward its modeling and simulation method based LPV model and MPC technology. In Section 1 of this paper, the identification and calculation strategy of LPV model are introduced; in Section 2, the modeling and simulation control method of circulation fluidized bed based on LPV model is discussed; Section 3 analyzes the simulation and control effectiveness; Section 4 is the conclusion.

\section{LPV MODEL IDENTIFICATION}

Recently, Zhu and Xu (2008) have developed a simple LPV model identification method. The method is described briefly for a multi-input single-output (MISO) process; for a multiinput multi-output (MIMO) process, the procedure can be repeated for each output. Let $y(t)$ denote the process output at discrete time $t, u(t)$ the input vector at time $t$. Denote variable $w(t)$ as the working-point variable (scheduling variable) which determines the working point of the process operation. Here $w(t)$ is a measured variable from the process or can be calculated from measured process variables:

$$
w(t) \in\left[w_{l o}, w_{h i}\right]
$$

where $w_{l o}$ and $w_{h i}$ are the low and the high limits of $w(t)$.

Assume that several, say three, linear models can be identified from corresponding working-points. Denote the three linear models at three working points as 


$$
\begin{array}{ll}
y^{1}(t)=\hat{G}_{1}^{1}(q) u_{1}(t)+\ldots+\hat{G}_{m}^{1}(q) u_{m}(t), & w(t)=w_{1} \\
y^{2}(t)=\hat{G}_{1}^{2}(q) u_{1}(t)+\ldots+\hat{G}_{m}^{2}(q) u_{m}(t), & w(t)=w_{2} \\
y^{3}(t)=\hat{G}_{1}^{3}(q) u_{1}(t)+\ldots+\hat{G}_{m}^{3}(q) u_{m}(t), & w(t)=w_{1}
\end{array}
$$

where $\hat{G}_{j}^{i}(q)$ is a linear transfer function, $q^{-1}$ is the unit delay operator, $m$ is the number of inputs and

$$
w_{l o} \leq w_{1}<w_{2}<w_{3} \leq w_{h i}
$$

Then the LPV model of the process based on the three linear models is given as model the process in the operatingtrajectory as follows

$$
\begin{aligned}
y(t)= & \alpha_{1}(w) y^{1}(t)+\alpha_{2}(w) y^{2}(t)+\alpha_{3}(w) y^{3}(t) \\
& =\alpha_{1}(w)\left[\hat{G}_{1}^{1}(q) u_{1}(t)+\ldots+\hat{G}_{m}^{1}(q) u_{m}(t)\right] \\
& +\alpha_{2}(w)\left[\hat{G}_{1}^{2}(q) u_{1}(t)+\ldots+\hat{G}_{m}^{2}(q) u_{m}(t)\right] \\
& +\alpha_{3}(w)\left[\hat{G}_{1}^{3}(q) u_{1}(t)+\ldots+\hat{G}_{m}^{3}(q) u_{m}(t)\right]+v(t)
\end{aligned}
$$

where $\alpha_{1}(w), \alpha_{2}(w)$ and $\alpha_{3}(w)$ are weights which are functions of the working point variable $w(t)$, the variable $v(t)$ is the unmeasured disturbance.

The procedure to identify the LPV model (2) consists of 2 steps:

Step 1: Identify the linear models in (1) using the data close to its corresponding working points. When the process can be tested at each working points, linear models can be easily obtained using a linear model identification method. When the working point variable varies continuously and no working-point test can be carried out, as in the case of the CFB boiler, then the test data need to be sliced into pieces and the data slices close to a working-point will be used to identify the corresponding linear model.

Step 2: Determine the weighting functions $\alpha_{1}(w), \alpha_{2}(w)$ and $\alpha_{3}(w)$. The easiest way to determine the weighting functions is to use so called triangular weights which is pre-assigned and need no estimation; another way is to use cubic splines as weights. In this case, data are needed to estimate the parameters and the data should contain both working point tests and transition tests; see Zhu and $\mathrm{Xu}$ (2008).

The LPV model using the triangular weights is given as:

$$
y(t)=\left\{\begin{array}{cc}
y^{1}(t) & w(t) \leq w_{1} \\
\frac{w_{2}-w(t)}{w_{2}-w_{1}} y^{1}(t)+\frac{w(t)-w_{1}}{w_{2}-w_{1}} y^{2}(t) & w_{1}<w(t) \leq w_{2} \\
\frac{w_{3}-w(t)}{w_{3}-w_{2}} y^{2}(t)+\frac{w(t)-w_{2}}{w_{3}-w_{2}} y^{3}(t) & w_{2}<w(t)<w_{3} \\
y^{3}(t) & w_{3} \leq w(t)
\end{array}\right.
$$

For cases where no working-point tests are permitted such as batch processes, Zhu and Ji (2009) have proposed a different model. Applying the weightings on the input side one obtains:

$$
\begin{aligned}
y(t) & =\left[\hat{G}_{1}^{1}(q) \alpha_{1}(w) u_{1}(t)+\ldots+\hat{G}_{m}^{1}(q) \alpha_{1}(w) u_{m}(t)\right] \\
& +\left[\hat{G}_{1}^{2}(q) \alpha_{2}(w) u_{1}(t)+\ldots+\hat{G}_{m}^{2}(q) \alpha_{2}(w) u_{m}(t)\right] \\
& +\left[\hat{G}_{1}^{3}(q) \alpha_{3}(w) u_{1}(t)+\ldots+\hat{G}_{m}^{3}(q) \alpha_{3}(w) u_{m}(t)\right]+v(t)
\end{aligned}
$$

Note that this model is in general different from (3). However, for slowly changing $w(t)$, the two models will be approximately equal. Moreover, pre-determined triangular weighting functions will be used in model (4). Therefore, the identification of the LPV model (4) using the pre-determined triangle weighting functions becomes a linear identification problem using multiple sets of weighted inputs.

\section{IDENTIFICATION OF A CFB BOILER}

In order to verify the usefulness of the simple LPV models (3) and (4) in modelling and control of industrial nonlinear processes, an industrial CFB boiler is used in the case study. The CFB boiler is a $35 \mathrm{t} / \mathrm{h}$ boiler and is located in Xiamen, China. This boiler uses coal as its main fuel, burning in the circulation fluidized bed, and its final product is steam. The boiler is basically controlled manually except that the steam drum level is controlled by a PID loop.

An MPC (model predictive control) project was initiated in order to realize the automatic control of the boiler. The main inputs (or manipulated variables, MVs) are coal feeder speed, primary air, secondary air, and fuel retain air; the main outputs (or controlled variables, $\mathrm{CVs}$ ) are furnace temperatures, furnace pressures, flue gas $\mathrm{O}_{2}$, steam temperature and steam pressure; the measured disturbance variable (DV) is the steam flow. The sampling time of the MPC controller was chosen as 30 seconds.

In order to generate informative data, identification tests have been carried out where test signals were added at the process inputs during normal operation. Generalized binary noise (GBN) signals (Zhu 2001, Chapter 3) are used as test signals and small amplitudes are used. The test lasted four days. The test signals do not disturb normal operation of the CFB boiler.

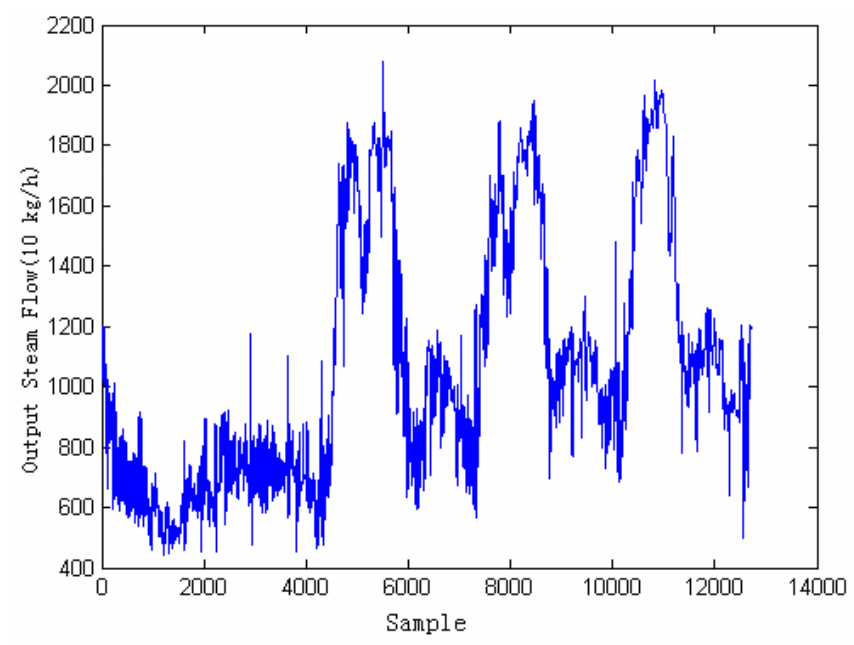

Fig.1 Steam flow during a 4 day period

\subsection{Working Point Variable}

The steam flow consumed by the users can change from $6 \mathrm{t} / \mathrm{h}$ at night to $25 \mathrm{t} / \mathrm{h}$ during day time; see Fig. 1. This huge 
fluctuation changes the combustion reaction in the furnace, and thus changes the behaviour of the process. So, the steam flow will be used as the working-point variable.

Using the steam flow as the working point variable, the production process is divided around two working points:

- low load working point at $w_{1}=8.5 \mathrm{t} / \mathrm{h}$

- high load working point at $w_{2}=16.4 \mathrm{t} / \mathrm{h}$

So the obtained LPV model will be the summation of two linear models.

\subsection{LPV Model Identification}

Denote the identified MIMO linear modes of the two working-points as:

$$
\begin{gathered}
\hat{y}^{1}=\left[\begin{array}{c}
\hat{y}_{1}(t) \\
\hat{y}_{2}(t) \\
\vdots \\
\hat{y}_{p}(t)
\end{array}\right]=\left[\begin{array}{ccc}
\hat{G}_{11} & \ldots & \hat{G}_{1 m} \\
\hat{G}_{21} & \ldots & \hat{G}_{2 m} \\
\ldots & \ldots & \ldots \\
\hat{G}_{p 1} & \ldots & \hat{G}_{p m}
\end{array}\right]\left[\begin{array}{c}
u_{1}(t) \\
u_{2}(t) \\
\vdots \\
u_{n}(t)
\end{array}\right] \quad w(t)=w_{1} \\
\hat{y}^{2}=\left[\begin{array}{c}
\hat{y}^{\prime}(t) \\
\hat{y}^{2^{\prime}}(t) \\
\vdots \\
\hat{y}^{\prime}(t)
\end{array}\right]=\left[\begin{array}{ccc}
\hat{G}_{11}^{\prime} & \cdots & \hat{G}_{1 m}^{\prime} \\
\hat{G}_{21}^{\prime} & \ldots & \hat{G}_{2 m}^{\prime} \\
\ldots & \ldots & \ldots \\
\hat{G}_{p 1}^{\prime} & \ldots & \hat{G}_{p m}^{\prime}
\end{array}\right]\left[\begin{array}{c}
u_{1}(t) \\
u_{2}(t) \\
\vdots \\
u_{n}(t)
\end{array}\right] \quad w(t)=w_{2}
\end{gathered}
$$

where $p$ is the number of controlled variables (CVs) and $m$ is the number of manipulated variables (MVs).

Then the LPV model (3) using the two working-points is given as

$$
\left[\begin{array}{c}
\hat{y}_{1}(t) \\
\hat{y}_{2}(t) \\
\vdots \\
\hat{y}_{p}(t)
\end{array}\right]=\alpha_{1}(w)\left[\begin{array}{c}
\hat{y}_{1}(t) \\
\hat{y}_{2}(t) \\
\vdots \\
\hat{y}_{p}(t)
\end{array}\right]+\alpha_{2}(w)\left[\begin{array}{c}
\hat{y}^{1^{\prime}}(t) \\
\hat{y}^{2^{\prime}}(t) \\
\vdots \\
\hat{y}^{\prime}(t)
\end{array}\right]
$$

where $\alpha_{1}(w)$ and $\alpha_{2}(w)$ are the triangular weighting functions:

$$
\begin{aligned}
& \alpha_{1}(w)=\left\{\begin{array}{cc}
1 & w(t)<8.5 \\
\frac{16.4-w(t)}{16.4-8.5} & 8.5 \leq w(t) \leq 16.4 \\
0 & w(t)>16.4
\end{array}\right. \\
& \alpha_{2}(w)=\left\{\begin{array}{cc}
0 & w(t)<8.5 \\
\frac{w(t)-8.5}{16.4-8.5} & 8.5 \leq w(t) \leq 16.4 \\
1 & w(t)>16.4
\end{array}\right.
\end{aligned}
$$

The data sequences of the four day test can be divided into seven parts or data slices according to the value of steam flow (Fig. 1), namely 4 slices are low load data and 3 slices are high load data. The low load data slices are used to identify the low load model (5); the high load data are used to identify the high load model (6); and finally the LPV model of the
CFB boiler is given as in (7). The linear models are identified using the ASYM method (Zhu, 2001, Chapters 7).

Not all the identified input/output models are used to form LPV model. When the disturbance level is high, some models have very poor quality and can have wrong signs in their gains. We ensure that the signs of the model gains agree with the process knowledge and when a model gain has the wrong sign, that model is set to zero.

Based on the process knowledge, one can determine the signs of model gains. The following corresponding relations were investigated:

- Relations between coal feeder speed and lower furnace temperature: if coal feeder speed increases, the bottoms furnace temperature would firstly decrease and then increase. Referring to the thermal principle, raw coal will absorb heat when it initially entered the furnace; then fuels begin to burn and release heat gradually, bringing furnace temperature higher.

- Relations between total air volume and furnace temperature: if total air volume increases, furnace temperature will firstly increase fast and then decrease slowly. Referring to the thermal principle, increasing air volume will increase oxygen concentration and speed up burning rate when it initially entered the furnace; for the air temperature is low and it will carry away the heat, finally, the furnace temperature will decrease.

- Relations between recycles and furnace temperature: if recycles increase, boiler furnace temperature will decrease, but the upper furnace temperature will increase. Referring to the thermal principle, increasing circulating materials will lead to decreasing combustion temperature, especially when the materials temperature is low.

- The changing trend of output steam temperature and upper furnace temperature are the same. Analyzing the boiler structure, heat exchangers are installed on upper boiler, so

\begin{tabular}{|c|c|c|c|}
\hline \multirow[b]{2}{*}{$\mathrm{CVs}$} & \multicolumn{3}{|c|}{ MVs (Gain of High/Low Load Model) } \\
\hline & $\begin{array}{l}\text { Primary } \\
\text { air }\end{array}$ & Retain air & $\begin{array}{c}\text { Coal } \\
\text { feeding }\end{array}$ \\
\hline Flue gas $\mathrm{O}_{2}$ & $2.19 / 1.75$ & $0.16 / 1.71$ & $0 / 0$ \\
\hline Steam pressure & $-0.5 / 0$ & $0.3 / 0.14$ & $0 / 0$ \\
\hline Steam temp & $-24.0 /-6.27$ & $25.0 / 17.6$ & $0.3 / 0.28$ \\
\hline $\begin{array}{l}\text { Furnace } \\
\text { pressure }\end{array}$ & $0.2 / 0$ & $0.1 / 0$ & $0 / 0$ \\
\hline $\begin{array}{l}\text { Furnace bott } \\
\text { temp }\end{array}$ & $8.8 / 17.9$ & $-13.9 /-9.4$ & $0.5 / 0.45$ \\
\hline
\end{tabular}
the change of upper furnace temperature will directly affect output steam temperature.

In table 1 we compare the gains of some models of high and low loads.

Table 1. Gain Comparison of High/Low Load Models

For comparison, a linear model was identified using the total data set. 
The LPV model and the linear model are simulated using the test data and the simulated outputs are compared to the measured outputs. In Fig. 2, four of the controlled variables: furnace pressure, steam pressure, furnace bottoms temperature and steam temperature, are plotted. One can see the LPV model outputs fit the measured outputs much better than those of the linear model.

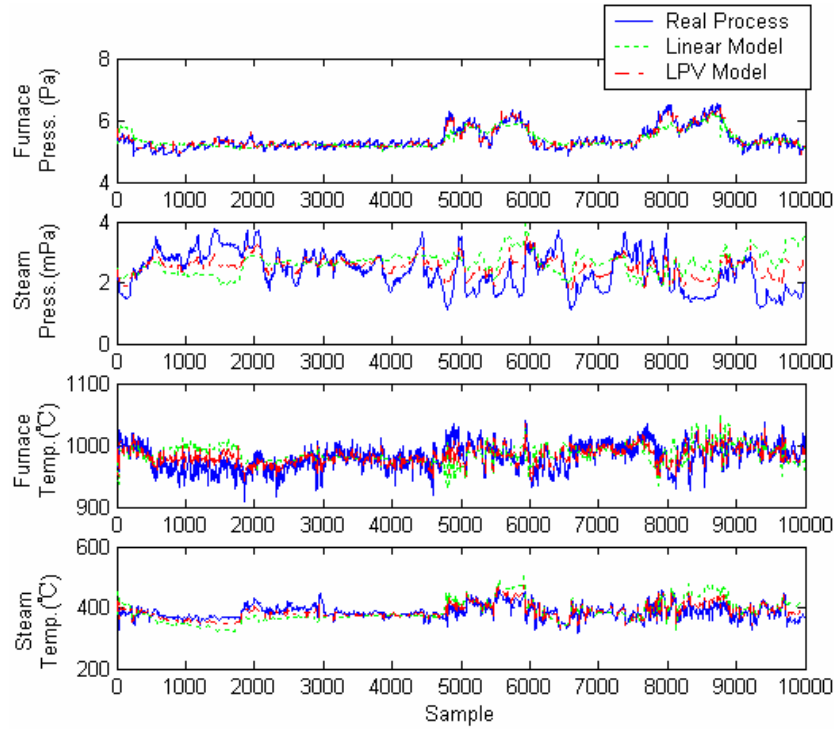

Fig. 2. Simulated outputs of linear model (green lines), of the LPV model (red lines) and measure outputs (blue lines)

\section{MPC CONTROL SIMULATIONS OF THE CFB BOILER}

Model predictive control (MPC) refers to a class of computer control algorithms that utilize an explicit process model to predict the future response of a plant. At each control interval an MPC algorithm attempts to optimize future plant behaviour by computing a sequence of future manipulated variable adjustments; see Qin and Badqwell (2003). Several studies of MPC for LPV model were proposed, a method based on a parameter-dependent control law was studied in Yu et.al. (2009), LEE and WON (2006) proposed a new robust MPC technique for LPV systems and nonlinear MPC based on the LPV model was proposed in Xu et.al. (2009) .In this paper, an MPC control algorithm was used to control CFB boiler. The algorithm consists of three steps: prediction, steady state optimization and dynamic control; see Zhu et.al. (2008). In the algorithm, if not enough degree of freedom is available, CV priorities and/or weightings will be used to resolve the conflict; when there are degree of freedom left after meet all CV control requirements, economic optimization will be performed. The economic optimization is realized by using combined LP (linear programming) and QP (quadratic programming):

$$
\begin{aligned}
& \min _{u}\left(\left\|u-I R V_{u}\right\|_{w_{u}}^{2}+\left\|y-I R V_{y}\right\|_{w_{y}}^{2}+b_{1}^{T} u+b_{2}^{T} y\right) \\
& \text { s.t. } \quad y=G u+d(t) \\
& \qquad y_{\min } \leq y \leq y_{\max } \\
& u_{\min } \leq u \leq u_{\max }
\end{aligned}
$$

Where $u$ is the vector of MVs, $y$ is the vector of $C V s, I R V_{u}$ is the vector of MV IRVs (ideal resting values), $I R V_{y}$ is the vector of CV IRVs, $w_{u}$ is the diagonal matrix of MV QP weightings, $w_{y}$ is the diagonal matrix of $\mathrm{CV}$ QP weightings, $b_{1}$ is the vector of MV LP weightings, $b_{2}$ is the vector of CV LP weightings, $G$ is the model gain matrix, $d(t)$ is the bias at sample time $t, y_{\min }$ and $y_{\max }$ are the vectors of CV low limits and high limits respectively, and $u \min$ and $u_{\max }$ are the vectors of MV low limits and high limits respectively.

The dynamic control part of the MPC algorithm uses the prediction values and process model to calculate the MV control actions that will drive the process to its steady state which is determined by the steady state optimization. The dynamic control calculation is again a QP:

$$
\begin{gathered}
\min _{u}\left[\begin{array}{r}
\sum_{j=1}^{P}\left\|y(t+j l t)-y^{r e f}(t+j)\right\|_{Q}^{2} \\
+\sum_{j=0}^{M-1}\left\|u(t+j)-u^{*}\right\|_{R}^{2} \\
+\sum_{j=0}^{M-1}\|\Delta U(t+j)\|_{S}^{2}
\end{array}\right] \\
\text { s.t. } \quad y^{r e f}(t+j)=y(t)+\left(y^{*}-y(t)\right)\left(1-e^{-j T / T_{\text {resp }}}\right) \\
u_{\min } \leq u(t+j) \leq u_{\max } \\
\Delta u_{\min } \leq \Delta u(t+j) \leq \Delta u_{\max }
\end{gathered}
$$

Where vector $y^{r e f}(t)$ is the vector of desired CV closed-loop response trajectories, vectors $y^{*}$ and $u^{*}$ are the steady state values of CVs and MVs respectively obtained by steady state optimization, $P$ is the prediction horizon, $M$ is the control horizon, $Q$ is the diagonal matrix of $\mathrm{CV}$ weightings, $R$ is the diagonal matrix of MV weightings, and $S$ is the diagonal matrix of MV increments weightings.

The LPV model plays two roles:

1) In the prediction part, the LPV model is used;

2) In the economic optimization (8) and dynamic control (9), the weights $w_{u}, w_{y}, Q, R$ and $S$ are no longer constants but functions of the working-point variable $w(t)$. First, these weights are determined at the same working points as the linear models (5) and (6). Then they are interpolated in the same way as the LPV model in (7).

Because the working-point variable steam flow is a disturbance variable (DV), the economic optimization (8) and dynamic control (9) can be solved using a QP which are the same as in linear MPC. However, if the working-point variable is an input (MV) or an output (CV), than a sequential-QP (SQP) should be used in (8) and (9).

The main control objectives are: 1) automate the operation; 2) stabilize the production; 3 ) reduced the coal consumption; and 4) reduce the NOx.

A software package has been developed that performs LPV model identification and MPC control using the LPV model. The LPV model of Section 3 was obtained using the software. Now the MPC control will be simulated using the software. In the simulation, the measured steam flow is used as the disturbance. In the simulation, the control objective is to keep important CVs as close to their setpoints as possible, namely to stabilize the operation. 
Simulated MPC controls using the LPV model and the linear model have been performed and their results are plotted in Fig. 3. One can see that the performance of the LPV model MPC is much higher than that of the linear model MPC. Fig.4 is the control signals of LPV model, and Table 2, Table 3 are the parameters of MPC control. Steam pressure and steam temperature are the most important CVs, we set their priority weight at 1 to ensure their control precision; a constant furnace bottom temperature guarantees safe production, so set its control strategy at "set point", that is to control its value as close to $980{ }^{\circ} \mathrm{C}$ as possible. The error weight and other weights of MVs and CVs can be calculated by their standard deviation and engineering experience.

Work is on the way to commission the developed MPC controller for the CFB boiler. In the above control objectives, the automatic operation of MPC has been achieved and control effect is good if the steam flow consumed by the users changes not great, but we have not got the data of coal consumption and NOx now. Because the great change of steam flow of the CFB boiler effects the precision of model and control, our next work is to increase the number of linear models which to be calculated LPV model. Results of simulation showed this method can efficiently reduce model output error. The economic optimization is also under consideration.
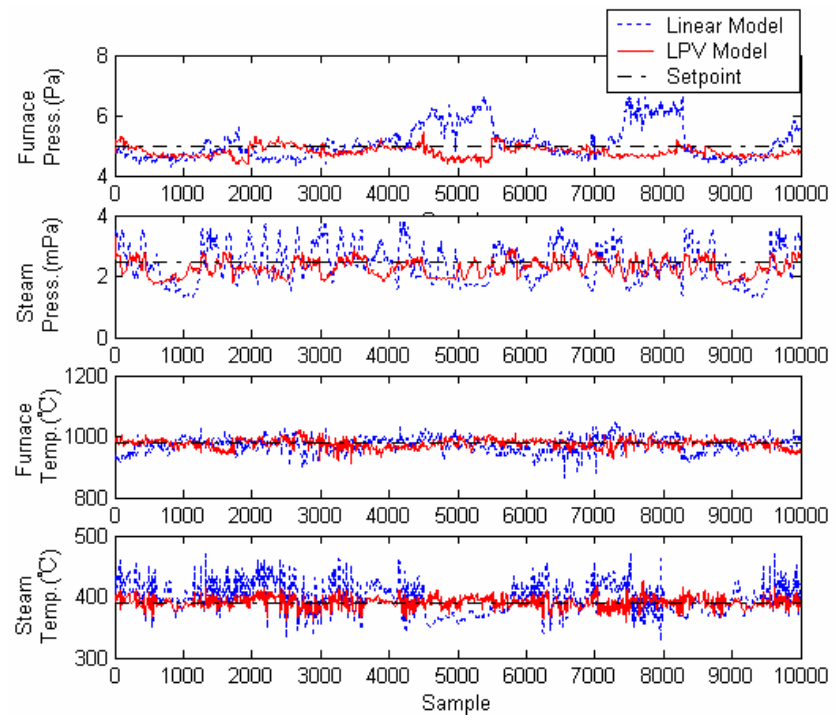

Fig.3 Simulation of MPC controllers using measured steam flow as disturbance (DV). CV setpoints are green lines; CVs of LPV MPC are red lines; CVs of linear MPC are blue lines

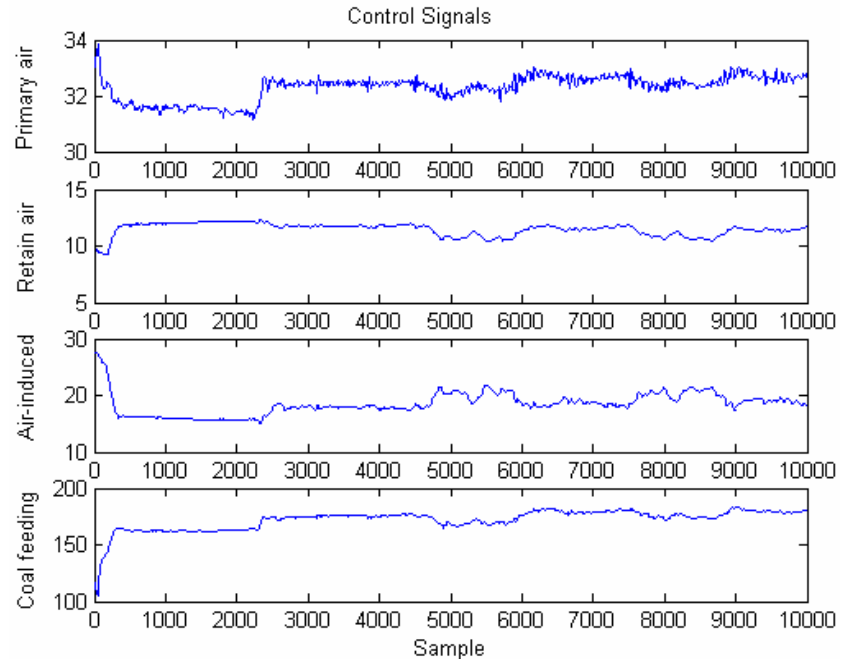

Fig. 4. Control signals

Table 2. Design of MPC Control for CVs

\begin{tabular}{|c|c|c|c|c|}
\hline CVs & $\begin{array}{c}\text { Control } \\
\text { strategy }\end{array}$ & $\begin{array}{c}\text { Set } \\
\text { point/Range }\end{array}$ & $\begin{array}{c}\text { Error } \\
\text { weight }\end{array}$ & $\begin{array}{c}\text { Priority } \\
\text { weight }\end{array}$ \\
\hline $\begin{array}{c}\text { Steam } \\
\text { pressure }\end{array}$ & Range & $2.2-2.6 \mathrm{mPa}$ & 1.56 & 1 \\
\hline $\begin{array}{c}\text { Steam } \\
\text { temp. }\end{array}$ & Range & $400-440{ }^{\circ} \mathrm{C}$ & 0.39 & 1 \\
\hline $\begin{array}{c}\text { Furnace } \\
\text { pressure }\end{array}$ & Range & $4.6-7.0 \mathrm{~Pa}$ & 5.07 & 2 \\
\hline $\begin{array}{c}\text { Furnace } \\
\text { bott. } \\
\text { temp }\end{array}$ & $\begin{array}{c}\text { Set } \\
\text { point }\end{array}$ & $980{ }^{\circ} \mathrm{C}$ & 0.5 & 2 \\
\hline
\end{tabular}

Table 3. Design of MPC Control for MVs

\begin{tabular}{|c|c|c|c|}
\hline MVs & $\begin{array}{l}\text { Increment } \\
\text { weight }\end{array}$ & IRV & Error weight \\
\hline Retain air & 0.7 & 8.89 & 0.7 \\
\hline $\begin{array}{l}\text { Air- } \\
\text { induced }\end{array}$ & 2.75 & 26.67 & 2.75 \\
\hline Primary air & 3.18 & 32.94 & 3.18 \\
\hline $\begin{array}{l}\text { Coal } \\
\text { feeding }\end{array}$ & 0.005 & 150 & 0.002 \\
\hline
\end{tabular}

\section{CONCLUSION}

This work reports an application of LPV model identification and MPC control to an industrial CFB boiler. Due to huge changes of steam load, the process shows strong nonlinearity. The use of a simple LPV model identification and MPC control has achieved much higher model accuracy and higher control performance than using a linear model. The case study shows that the LPV model has good approximation power in modelling industrial processes and it is easy to use in MPC control. Batch processes often show strong nonlinearity. The authors believe that the LPV models can be used for the identification and control of batch processes, for a batch process, its operating trajectory is its recipe or operation curve carried out to produce a product. 


\section{ACKNOWLEDGMENT}

This research was supported by Key Science and Technology Plan Project of Fujian Province (No. 2009H0044), National Natural Science Foundation of Fujian Province (No. 2009J1303) and Xiamen University national 2113 rd period project (No.0630-E62000).

\section{REFERENCES}

Dos Santos, P. L., J. A. Ramos, \& J. L. M. de Carvalho (2007). Identification of linear parameter varying systems using an iterative deterministic-stochastic subspace approach. Proceedings of the European Control Conference, pp. 4867-4873.

Lee, S and S. Won (2006). Model predictive control for linear parameter varying systems using a new parameter dependent terminal weighting matrix. IEICE Transactions on Fundamentals of Electronics, Communications and Computer Sciences, Vol. E89A,pp. 2166-2172.

Murray-Smith, R., \& T. A. Johansen (1997). Multiple Model Approaches to Modeling and Control. Taylor and Francis.

Roland Tóth, P.S.C. Heuberger and P.M.J. Van den Hof (2009). Asymptotically optimal orthonormal basis functions for LPV system identification. Automatica, Vol. 45, pp. 1359-1370.

Rugh, W.J. and J. Shamma (2000). Research on gain scheduling. Automatica, Vol. 36, pp. 1401-1425.

Shamma, J. and M. Athans (1991). Guaranteed properties of gain scheduled control for linear parameter varying plants. Automatica, Vol. 27, pp. 559-564.

Qin, S.J. and T.A. Badgwell (2003). A survey of industrial model predictive control technology. Control Engineering Practice, Vol. 11, pp. 733-764.

$\mathrm{Xu}$, Z.H., J. Zhao, J.X. Qian, Y.C. Zhu (2009). Nonlinear MPC using an Identified LPV Model. Ind. Eng. Chen. Res., Vol.48, pp. 3043-3051.

Yu, S.Y., C. Bohm, H. Chen, F.Allgower (2009).Stabilizing Model Predictive Control for LPV Systems Subject to Constraints with Parameter-Dependent Control Law. 2009 American Control Conference, St. Louis, MO, USA, June 10-12, 2009.

Zhu, Y.C. (2001). Multivariable System Identification for Process Control. Elsevier, London.

Zhu, Y.C., Z.H Xu, J. Zhao, K. Han, J.X. Qian, \& W.X. Li (2008). Development and Application of an Integrated MPC Technology. Preprint of IFAC World Congress, July 6-11, 2008, Seoul, Vol. 17, pp. 6962-6967.

Zhu, Y.C. and Z.H. Xu (2008). A method of LPV model identification for control. Preprint of IFAC World Congress, July 6-11, 2008, Seoul, Vol.11, pp. 50185023.

Zhu, Y.C. and G.L. Ji (2009). LPV model identification using blended linear models with given weightings. Preprint of IFAC Symposium of System Identification, July 6-8, 2009, San Malo, pp. 1674-1679. 